\title{
Recent trends in mortality due to testicular cancer in Ireland: a comparison with England and Wales
}

\author{
JOHN A THORNHILL, RONAN M CONROY, DANIEL G KELLY, ANTHONY \\ WALSH, JAMES J FENNELLY AND JOHN M FITZPATRICK \\ From The Irish Testicular Tumour Registry, 5 Northumberland Road,.Dublin 4, Ireland
}

SUMMARY In the period 1961-84, the number of deaths in Ireland due to testis cancer rose by $64 \%$. This was due both to significant male population expansion $(25 \cdot 3 \%)$ and to an increased mortality rate. In the 25-34 years age group, one of the groups at highest risk, the mortality rate rose by $123 \%$. In contrast, in England and Wales, although the male population has risen by $8.5 \%$ since 1961 , the number of deaths has decreased by $17 \%$. This is due to falling mortality rates, for example an $18 \%$ decrease in those aged 25-34 years. The highest rate of all occurred in those Irish over 75 years old. The changing Irish trends appear to lag behind those in England and Wales by some decades, and this raises important aetiological considerations.

Testis tumours are rare, accounting for approximately $1 \%$ off all male neoplasms. ${ }^{1}$ However, the incidence is reported to be increasing in several countries. ${ }^{2}$ This trend is particularly marked in Denmark ${ }^{3}$ whose annual rate of 8-9 per $10^{5}$ males is much greater than in other countries where the annual incidence is generally low and between 2 and 3 per $10^{5}$ male population. ${ }^{4}$

The importance of a rising incidence is underlined by two factors particularly rilevant to this type of cancer. Firstly, traditional figures show that this disease affects men at crucial ages, that is, predominantly in the $20-40$ years age group. ${ }^{5}$ It is now the fourth commonest cause of death in males aged 15-34 years ${ }^{6}$ and the leading cause of lethal cancer between 25 and 34 years, ${ }^{7}$ a time of life when not only is work productivity often high but likewise the number of dependents and financial and social commitments. Secondly, while one male in 500 will get the disease before the age of 50 and approximately $25 \%$ of those affected will die as a result, there is little doubt that multidisciplinary management in a specialist setting can now hope for a nearly $100 \%$ cure rate in early stage disease and almost $75 \%$ cure rate overall. Similar results are not achieved in any other solid tumour in males. ${ }^{7}$ This is because of major clinical advances, especially in the fields of chemotherapy, ${ }^{9}$ serum tumour markers ${ }^{10}$ and coordinated treatment. ${ }^{11}$

There has previously been no study of Irish incidence or mortality rates although reference has been made to possible rising figures. ${ }^{12}$ This investigation was therefore undertaken to obtain a comparative estimate of the magnitude of the problem of testicular cancer in Ireland, England and Wales and to gain a better insight into current trends in all regions.

\section{Methods}

Mortality data for Ireland were supplied by the Department of Health and the Central Statistic Office, and for England and Wales by the Office of Population Censuses and Surveys. The distribution of the male population at risk was calculated from supplied figures.

Both mortality and demographic data were analysed in three octennia: 1961-68, 1969-76, and 1977-84. Because of the rarity of this disease, deaths were pooled in ten-year age groups instead of the usual five. Likewise, to ensure meaningful reporting, children up to age 15 years and those older than 75 years were considered single groups.

To verify any trends in mortality rates, deaths were analysed by birth cohort. Cohorts born at extremes (eg, 1900 and 1959) are not directly comparable because of their wide age difference during the 24 year study period. In those born in interim years, however, there is good crossover, and within these limitations all data can be considered an accurate reflection of actual mortality rates.

To verify findings, it was considered necessary to test the reliability of the basic mortality data. All relevant Irish death certificates in the years 1980-84 
were individually scrutinised, and registry material for the same period was compared.

\section{Results}

The number of deaths due to testicular cancer in Ireland increased from 73 in 1961-68 to 120 in $1977-85$, an increase of $64 \%$. There was an increase of $25.3 \%$ in the male population over the 24 years. In contrast, the corresponding deaths in England and

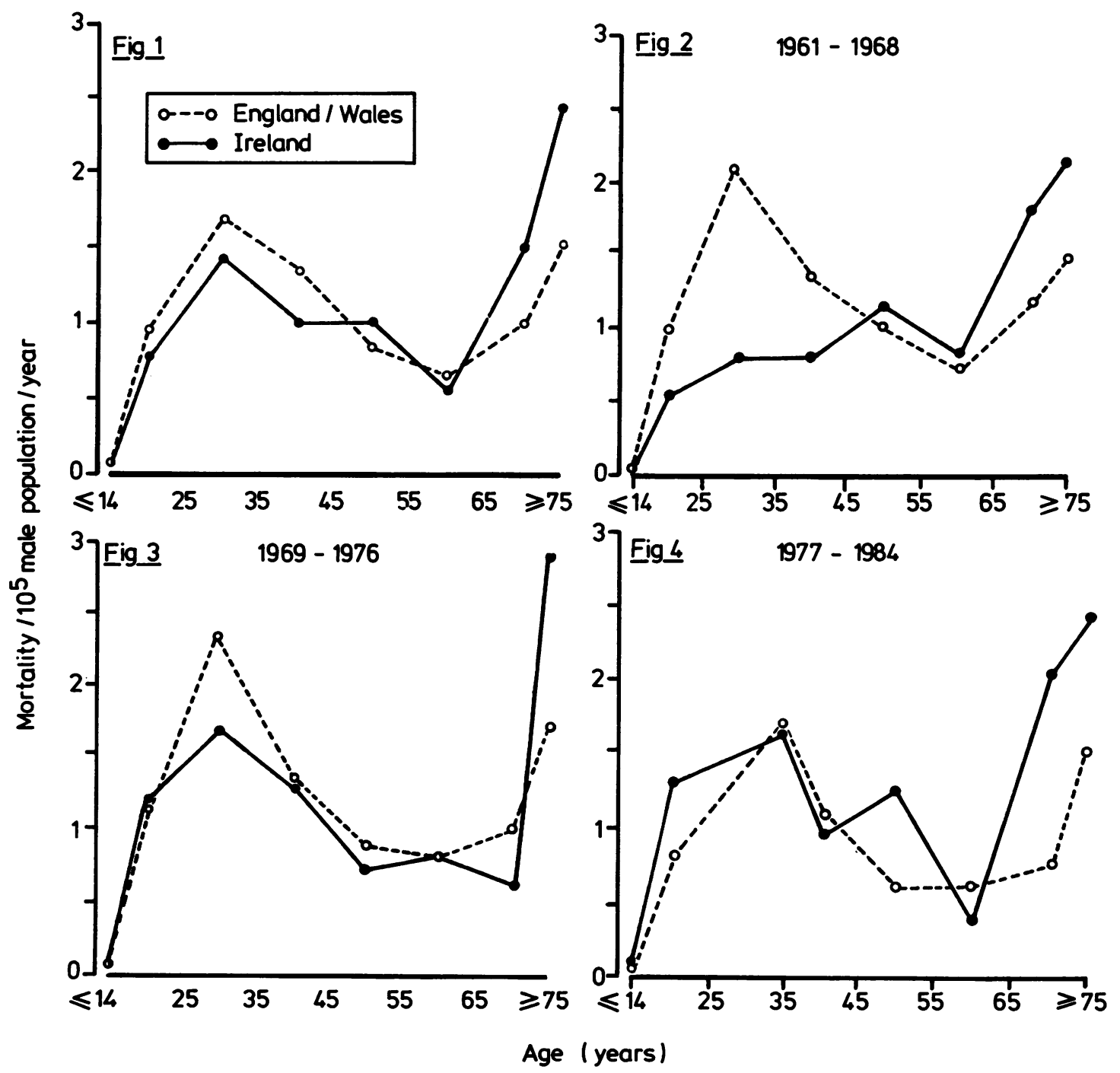

Fig 1 Comparative age specific mortality rates from testis cancer in Ireland and England/Wales 1961-84.

Fig 3 Comparative age specific mortality rates in Ireland and England/Wales, 1969-76.
Wales decreased from 1736 to 1486 , a reduction of $16.8 \%$ while the male population increased by $8.5 \%$.

The age distribution of deaths and mortality rates in the period 1961-84 showed a peak at ages 25-34 years for all regions (fig. 1). In England and Wales the distribution was bimodal with moderate levels also seen in the elderly. The equivalent Irish rates showed a lesser peak in young adults, and the highest age specific rates of all occurred in Ireland in those over 75 years old.

Fig 2 Comparative age specific mortality rates in Ireland and England/Wales, 1961-68.

Fig 4 Comparative age specific mortality rates in Ireland and England/Wales 1977-84. 
Comparative trends in age specific mortality rates over the 24 years are illustrated (figs 2 to 4 ). There has been a marked increase in Irish rates over the period: 0.5 to 1.3 per $10^{5}$ in ages $15-24$ years, an increase of $156 \% ; 0.75$ to 1.77 in ages $25-34$ years, an increase of $123 \%$; and $2 \cdot 11$ to 2.39 in those older than 75 years, an increase of $13 \%$. In contrast, rates in England and Wales have decreased in all age groups except the very elderly; 0.97 to 0.94 per $10^{5}$ in ages $15-24$ years, a decrease of $3 \% ; 2.08$ to 1.70 in ages $25-34$ years, a decrease of $18 \%$; and 1.40 to 1.50 in those over 75 years, an increase of $7 \%$.

The end result of these opposing trends in mortality has been that the rates for ages 15-34 years in all regions are now very similar. This is in sharp contrast to the situation two decades ago. The relative very high rate in Ireland in those over 75 years has been consistently at variance with equivalent rates in the other countries. Age distribution has remained bimodal in England and Wales with dimishing accentuation whereas the trimodal character of Irish figures has developed and become more pronounced.

The mortality rates of ten year birth cohorts in Ireland are shown in figure 5. These confirm the rising trend in those aged $20-45$ years, and show a consistent low incidence in those of middle age, while the evidence of a rising rate in old age is not outruled. Cohort rates for England and Wales (not illustrated) did not show significant alteration during the study

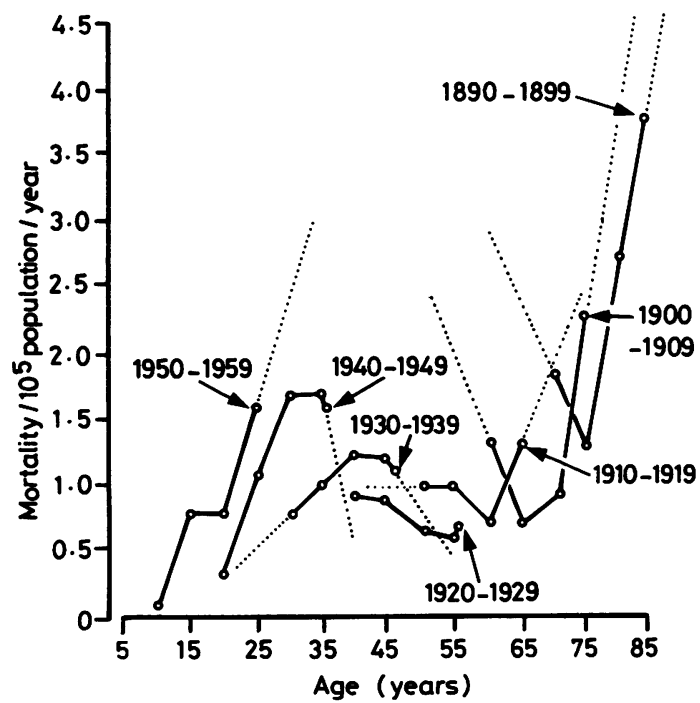

Fig 5 Age specific mortality rates for male ten-year cohorts in Ireland.*

* Results are accurate to the limits of the extrapolations shown. period in accord with trends in actuarial mortality rates.

Inspection of the relevant Irish death certificates between 1980 and 1984 and comparison with equivalent registry material revealed some discrepancy in mortality data. One case $(1 \cdot 3 \%$ of total in period) of prostatic cancer was incorrectly coded as a testis cancer on the death certificate. Two cases $(2.7 \%$ of total) were not germ-cell tumours; one leiomyosarcoma of old age and one rhabdomyosarcoma in a young adult. The registry also revealed one mortality in which testis cancer had not been entered on the death certificate when this may have been appropriate.

\section{Discussion}

The significance of the increasing Irish mortality rate, especially in the 15-44 years age group, is emphasised by study of the relevant subpopulation at risk. Its continued remarkable expansion, by $51 \%$ over 24 years, can be expected to yield an ever increasing number of cases in future decades. In England and Wales, a similar but much less marked demographic trend had a less significant effect on the number of deaths that occurred. Their combination of moderate population growth $(8.4 \%$ in age group $15-44$ years over 24 years) with falling mortality rates should ensure a continued decrease in the number of deaths from this disease.

The causes of increasing Irish rates in the 15-44 age group remain unexplained. The study of mortality data normally excludes details of histological classification but this study confirmed that nearly all tumours were of germ cell origin. Some studies have shown the incidence of teratomas to be rising more rapidly than seminomas ${ }^{13,14}$ and this phenomenon could explain the trend towards greater increases in younger age groups, confirmed by cohort studies. This theory is supported by the emergence of a lesser peak in mortality rates in ages $45-54$ years, which could be specifically due to seminomas. Although the aetiology of testicular cancer remains obscure, the accepted predisposing factors of maldescent, unilateral atrophy with infertility and ambiguous $\operatorname{sex}^{15}$ are unlikely to have altered over the study period. It is therefore likely that other causative factors were active. Better awareness and improved methods of diagnosis may, by increasing detection of testicular cancer, play a role in increasing apparent numbers; but, in contrast, recent advances in management should have reduced mortality rates. Major improvements in treatment results have been documented in England and Wales, ${ }^{16,17}$ and this could partially explain the falling rate. An evaluation of Irish treatment results is awaited but it is unlikely that the above factors, even if 
remarkably at variance between the countries, could explain the rising Irish rates or the disparity in national trends.

The relative very high rate in old age in Ireland was unexpected. It was consistently greater than in England and Wales, and likewise the Irish rate of increase was nearly double that of the other countries. The possibility that such rates were artificially elevated by including cases of lymphoma was not supported by examination of individual death certificates. Results from other countries have shown a similar high incidence in old age many decades ago. ${ }^{18}$ It remains to be seen whether present Irish trends continue; this has not been observed in other studies ${ }^{19,20}$ where rates in old age have diminished concomitantly with the large increase in deaths in young males. It is attractive to propose that later Irish cohorts will not in future have high rates when elderly because they will have already suffered the disease at an earlier age.

The trends in Irish mortality rates have radically altered the shape of the age specific curve. The most recent graphs of the three countries are at least similar, but the Irish graph for 1961-68 had little in common with the equivalent for the other regions: in fact, it closely resembled the shape of the graph for England and Wales in the year 1911. ${ }^{21}$ Specific increases in mortality rates are known to have occurred in England and Wales after 1911 so that the shape of the present curve was first evident in figures for 1951. This strongly suggests that similar changes in mortality have occurred in all the regions but that the Irish trends may lag behind those of England and Wales by some decades. The reasons for such an apparent time lag between the countries has important implications concerning the local aetiology of the disease. One may postulate that causative factors, present in England and Wales some time ago, became active in Ireland only after subsequent years. Perhaps environmental differences such as the later industrialisation of Ireland are relevant. Further epidemiological studies are needed in the search to explain the hitherto unkown aetiology of this disease.

\section{Conclusion}

Recent trends in mortality due to testis cancer in Ireland are in marked contrast to those in England and Wales. Results from the latter are encouraging but the Irish figures are a warning of a continued dramatic increase in future decades. The discovery of large differences in some age specific rates, opposing trends, and a time lag between the countries underlines the need to explain the aetiology of the disease. The importance of continued future planning to ensure maximum nationwide disease control is emphasised.
The authors wish to thank Miss V Freeman for typing the manuscript.

The Irish Testicular Tumour Registry is funded by grant from The Irish Cancer Society.

Address for reprints: Mr John A. Thornhill, The Irish Testicular Tumour Registry, 5 Northumberland Road, Dublin 4.

\section{References}

${ }^{1}$ Droller MJ. Cancer of the testis: An overview. Urol Clin Nth Amer 1980; 7: 731-3.

2 Waterhouse J, Muir C, Shanmugaratnum K, Powell J. Cancer incidence in five continents Vol. IV. IARC Scient Publications 1982: 42.

${ }^{3}$ Schultz HP, Arends J, Barlebo $\mathrm{H}$, et al. Testicular carcinoma in Denmark 1976-1980. Acta Radiol Oncol 1984: 23: 249-61.

${ }^{4}$ Ekman P. Current concepts in the management of testicular germ cell tumours in adults. Int J Androl 1982: 5: 356-66.

${ }^{5}$ Friedman NB, Moore RA. Tumors of the testis: Report on 922 cases. Milit Surgeon 1946: 99: 573-93.

6 Mostofi FK. Testicular tumours-epidemiology, etiology and pathologic features. Cancer 1973: 32: 1186.

${ }^{7}$ McKay EN, Sellers HH. A statistical review of malignant testicular tumors based on the experience of the Ontario Cancer Foundation clinics, 1983 to 1961. Can Med Assoc J 1966: 94: 889-99.

${ }^{8}$ Oliver RTD. Testicular germ cell tumours - a model for a new approach to treatment of adult solid tumours. Postgrad Med J 1985: 61: 123-31.

${ }^{9}$ Newlands ES, Begnet RHJ, Rustin GJS, Bagshawe KD. Further advances in the management of malignant teratomas of the testis and other sites. Proceedings of the 13th International Congress of Chemotherapy, Vienna. Part 241: 7-9.

10 Ostrow SS. The management of nonseminomatous testicular cancer: current therapy and future directions. Amer J Med Sci 1983: 285: 24-37.

11 Einhorn LH. Testicular cancer as a model for a curable neoplasm: The Richard Hinda Rosenthal Foundation award lecture. Cancer Research 1981: 41: 3275-80.

12 Kelly DG. Testicular tumours: proposals for a plan of management and Tumour Registry. Irish Med J 1983: 76: 216-7.

${ }^{13} \mathrm{Krag}$ Jacobsen G, Barlebo H, Olsen J, et al. Testicular germ cell tumours in Denmark 1976-1980. Pathology of 1058 consecutive cases. Acta Radiol Oncol 1984: 23: 239-47.

14 Waterhouse JAH. Epidemiology of testicular tumours. $J$ Roy Soc Med 1985: 78: Suppl 6: 3-7.

15 Anonymous. Early testicular cancer. Lancet 1980: 2: 1175.

16 Newlands ES, Rustin GJS, Begent RJHJ, Parker D, Bagshawe KD. Further advances in the management of malignant teratomas of the testis and other sites. Lancet 1983. 948-951.

${ }^{17}$ Peckham MJ, Barrett A, Liew KH, et al. The treatment of metastatic germ cell testicular tumours in the bleomycin, etoposide and cis-platin (BEP) Br J Cancer 1983: 47: 613-9. 
${ }^{18}$ Grumet RF, MacMahon B. Trends in mortality from neoplasms of the testis. Cancer 1958: 11: 790-7.

${ }^{19}$ Peterson GR, Lee JAH. Secular trends of malignant tumours of the testis in white men. J Nat Cancer Inst 1972: 43: 339-54.
${ }^{20}$ Schottenfield D, Warshawer ME, Sherlock S, Zanber AG, Leder M, Payne R. The epidemiology of testicular cancer in young adults. Amer J Epidemiol 1980: 112: 232-46.

${ }^{21}$ Davies JM. Testicular cancer in England \& Wales: Some epidemiological aspects. Lancet 1981: Apr: 928-32. 